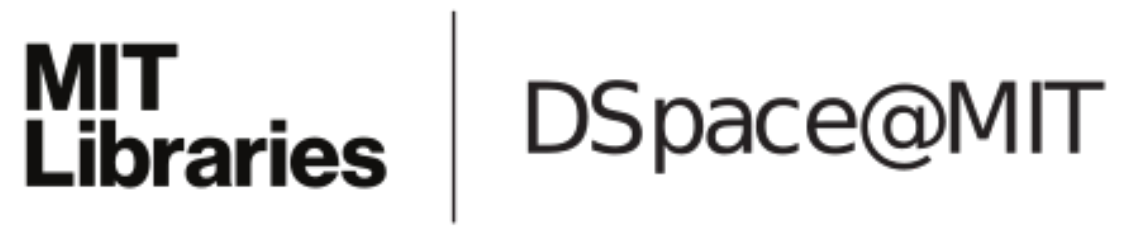

\author{
MIT Open Access Articles
}

Enhanced current-induced domain wall motion by tuning perpendicular magnetic anisotropy

The MIT Faculty has made this article openly available. Please share how this access benefits you. Your story matters.

Citation: Emori, Satoru, and Geoffrey S. D. Beach. Enhanced Current-induced Domain Wall Motion by Tuning Perpendicular Magnetic Anisotropy. Applied Physics Letters 98, no. 13 (2011): (C) 2011 American Institute of Physics

As Published: http://dx.doi.org/10.1063/1.3570652

Publisher: American Institute of Physics (AIP)

Persistent URL: http://hdl.handle.net/1721.1/79699

Version: Final published version: final published article, as it appeared in a journal, conference proceedings, or other formally published context

Terms of Use: Article is made available in accordance with the publisher's policy and may be subject to US copyright law. Please refer to the publisher's site for terms of use. 


\section{AIP Applied Physics \\ Letters}

\section{Enhanced current-induced domain wall motion by tuning perpendicular magnetic anisotropy}

Satoru Emori and Geoffrey S. D. Beach

Citation: Appl. Phys. Lett. 98, 132508 (2011); doi: 10.1063/1.3570652

View online: http://dx.doi.org/10.1063/1.3570652

View Table of Contents: http://apl.aip.org/resource/1/APPLAB/v98/i13

Published by the American Institute of Physics.

\section{Additional information on Appl. Phys. Lett.}

Journal Homepage: http://apl.aip.org/

Journal Information: http://apl.aip.org/about/about_the_journal

Top downloads: http://apl.aip.org/features/most_downloaded

Information for Authors: http://apl.aip.org/authors

\section{ADVERTISEMENT}
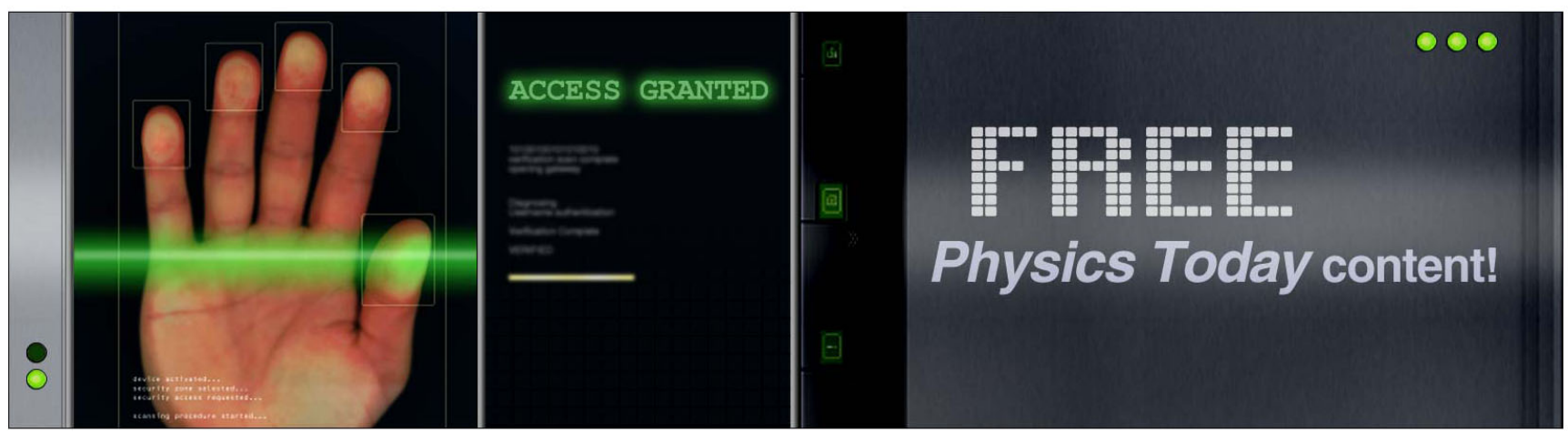


\title{
Enhanced current-induced domain wall motion by tuning perpendicular magnetic anisotropy
}

\author{
Satoru Emori ${ }^{\text {a) }}$ and Geoffrey S. D. Beach \\ Department of Materials Science and Engineering, Massachusetts Institute of Technology, Cambridge, \\ Massachusetts 02139-4307, USA
}

(Received 1 December 2010; accepted 5 March 2011; published online 31 March 2011)

\begin{abstract}
The effect of perpendicular magnetic anisotropy (PMA) on current-induced domain wall (DW) motion is investigated by micromagnetic simulations. The critical current density $J_{C}$ to drive DWs into periodic transformation and continuous motion by adiabatic spin transfer torque decreases with increasing PMA. Also, with optimized PMA that almost exactly compensates the demagnetizing field, the adiabatic displacement of DWs driven by currents less than $J_{C}$ is strongly enhanced. Since PMA can be controlled easily in magnetic multilayer films, this technique of enhancing current-induced DW motion may be practical for device applications. (C) 2011 American Institute of Physics. [doi:10.1063/1.3570652]
\end{abstract}

Electric current can move domain walls (DWs) in submicron-wide ferromagnetic strips through spin transfer torque, arising from the exchange of angular momentum between spin-polarized electrons and DW magnetic moments. ${ }^{1-5}$ Such current-induced DW motion has become an active area of research to develop novel spintronic RAM, ${ }^{6}$ data storage, ${ }^{7}$ and logic devices. ${ }^{8}$ However, the critical current density $J_{C}$ required to move a DW remains too large for practical applications.

In the one-dimensional model of current-induced DW motion, $J_{C}$ is proportional to the hard axis anisotropy $K_{\perp}$ of the ferromagnetic strip. ${ }^{2}$ A DW must overcome $K_{\perp}$ to precess and move continuously in the direction of the spinpolarized electron current. A variety of experimental and computational studies have reduced $J_{C}$ by minimizing $K_{\perp}$. For in-plane magnetized strips such as those of permalloy with small magnetocrystalline anisotropy, $K_{\perp}$ is the difference between the out-of-plane and transverse demagnetizing constants $\left|K_{z}-K_{y}\right|$. A systematic reduction in $J_{C}$ in permalloy strips was observed by decreasing $K_{\perp}$ through the control of the cross-sectional aspect ratio (width over thickness). ${ }^{9,10}$ Lower $J_{C}$ values have also been exhibited by out-of-plane magnetized strips with strong perpendicular magnetic anisotropy (PMA), ${ }^{6,11-13}$ whose $K_{\perp}$ is the difference between the transverse and longitudinal demagnetizing constants $\mid K_{y}$ $-K_{x} \mid$. For out-of-plane magnetized strips, $K_{\perp}$ (already smaller than $K_{\perp}=\left|K_{z}-K_{y}\right|$ for typical in-plane magnetized strips) can be reduced further by tuning their cross-sectional aspect ratio. ${ }^{6,11,12}$ Furthermore, recent theoretical studies have shown reductions in $J_{C}$ for cylindrical nanowires (in which $K_{\perp}$ goes to zero by symmetry) ${ }^{14}$ and strips with Dzyaloshinskii-Moriya interaction (in which built-in spiral DW configurations reduce $\left.\left.K_{\perp}\right)\right)^{15}$

This letter presents an alternative means of enhancing current-induced DW motion by adjusting PMA in easily tunable material systems such as multilayers of $\mathrm{Co} / \mathrm{Pt} .{ }^{16}$ In a strip with enough PMA to balance out-of-plane demagnetization, $K_{\perp}$ is minimized and $J_{C}$ is reduced. Moreover, in strips with optimized PMA, DW displacement at current below the threshold for continuous motion is strongly en-

${ }^{a)}$ Electronic mail: satorue@mit.edu. hanced, which may be useful for devices with engineered pinning sites.

The effect of PMA on current-induced DW motion in a more realistic two-dimensional framework is calculated using the public OOMMF micromagnetic simulation code. ${ }^{17}$ Zero-temperature simulations were performed on a series of strips with various PMA "quality factors" ${ }^{18} Q=K_{U} / 2 \pi M_{S}^{2}$, where $K_{U}$ is the uniaxial PMA constant and $2 \pi M_{S}^{2}$ is the demagnetizing energy. Material parameters of the strips were those of $\mathrm{Co}$, which approximate typical parameters for $\mathrm{Co} / \mathrm{Pt}$ (exchange constant $A=2 \times 10^{-6} \mathrm{erg} / \mathrm{cm}$, saturation magnetization $M_{S}=1400 \mathrm{emu} / \mathrm{cm}^{3}$, Gilbert damping parameter $\alpha=0.03$ ). As the PMA of $\mathrm{Co} / \mathrm{Pt}$ multilayers is inversely related to the thickness of the Co layer $t_{\mathrm{Co}}$, we model the system with a fixed surface anisotropy constant $K_{S}$ $=0.585 \mathrm{erg} / \mathrm{cm}^{2}$ and set $K_{U}=2 K_{S} / t_{\mathrm{Co}}{ }^{16}$ The width of each strip was held constant at $120 \mathrm{~nm}$, unless otherwise specified. The xy cell size was $4 \times 4 \mathrm{~nm}^{2}$ for most samples $(2.5$ $\times 2.5 \mathrm{~nm}^{2}$ for $Q=1.58,2 \times 2 \mathrm{~nm}^{2}$ for $Q=2.38$ ), and the cell thickness was set to $3 t_{\mathrm{Co}}$. The simulated length of the strip was $6 \mu \mathrm{m}$ with fixed magnetization on both ends.

As shown in Fig. 1, transverse DWs and Bloch DWs were initialized in the middle of in-plane magnetized strips $(Q<1)$ and out-of-plane magnetized strips $(Q>1)$, respectively. As $Q$ approaches 1 for in-plane magnetized strips [see Fig. 1(c) with $Q=0.95]$, the magnetization cants out of the plane along the strip edges with opposite orientation on either edge. This out-of-plane canting is reminiscent of stripe domain formation in continuous films with $Q \approx 1$. By con-

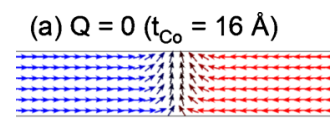

(c) $\mathrm{Q}=0.95\left(\mathrm{t}_{\mathrm{Co}}=10 \AA\right)$

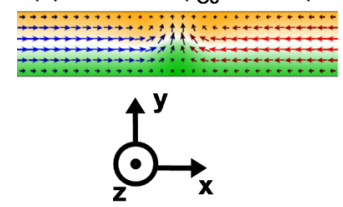

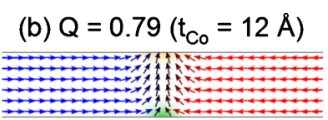

(d) $Q=1.06\left(t_{C_{0}}=9 \AA\right)$

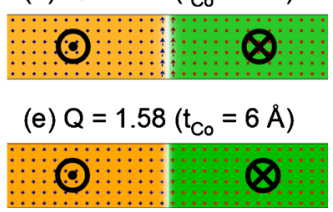

FIG. 1. (Color online) Initialized transverse [(a)-(c)] and Bloch [(d) and (e)] DWs with different PMA quality factors. 
trast, just above the anisotropy threshold, the strip with $Q$ $=1.06[$ Fig. $1(\mathrm{~d})]$ contains a well-defined narrow Bloch DW with no apparent spontaneous formation of a stripelike configuration. As $Q$ increases beyond 1, the Bloch DW becomes slightly narrower [Fig. 1(e)].

These DWs were subjected to field- and current-driven simulations, governed by the Landau-Lifshitz-Gilbert equation at zero temperature with an added term for adiabatic spin transfer torque. ${ }^{3,19}$ A major aim of these micromagnetic simulations was to correlate the onset of field-driven precessional motion and current-driven continuous motion. Above a threshold applied field, known as the Walker breakdown field $H_{W}$, a DW moves by transforming periodically (between transverse and antivortex for $Q<1$, between Bloch and Néel for $Q>1)^{4,5}$ analogous to the precessional motion in the one-dimensional model. In the adiabatic limit of the current-driven case, a DW can move continuously only if its configuration can be transformed by spin torque strong enough to overcome $K_{\perp}$. Thus, a DW driven by current also moves by periodic transformation, similar to the field-driven motion above the Walker breakdown. Suppressing $K_{\perp}$ by tuning PMA should facilitate the onset of both the fielddriven Walker breakdown and current-driven motion of DWs.

Field-driven simulations [some of which are shown in Fig. 2(a)] were performed with zero current (spin-polarized electron velocity $u=0),{ }^{20}$ and a uniform magnetic field was applied in the longitudinal $(+x)$ direction of each strip with $Q<1$, and in the out-of-plane $(+z)$ direction of each strip with $Q>1$. For each sample, the average DW velocity reaches a maximum $v_{W}$ when the applied field is $H_{W}$. Above $H_{W}$, the average DW velocity drops abruptly as the DW overcomes $K_{\perp}$ and propagates by changing its configuration periodically. Since PMA reduces $K_{\perp}$, DWs in strips with greater $Q$ breakdown at lower $v_{W}$. In current-driven simulations [Fig. 2(b)], the spin-polarized electron current was injected in the $+x$ direction. Each sample exhibits a critical current $u_{C}\left(\sim J_{C}\right)$ below which the average DW velocity is 0 . Similar to $v_{W}, u_{C}$ decreases as larger $Q$ facilitates DW motion by periodic transformation.

Figure 2(c) summarizes the decrease in both $v_{W}$ and $u_{C}$ with the magnitude of PMA. The close overlap of the $v_{W}$ and $u_{C}$ curves implies that the DW begins to move continuously when the effective electron velocity $u$ reaches $v_{W}$, qualitatively consistent with the simple one-dimensional model of adiabatic spin transfer. The remarkable decrease in both $v_{W}$ and $u_{C}$ as $Q$ increases from 0 to 1 is due to the drastic decrease in $K_{\perp}=\left|K_{z}-K_{y}\right|$, where $K_{z} \approx 2 \pi M_{S}^{2}-K_{U}$. At $Q \approx 1$, the uniaxial PMA constant $K_{U}$ cancels out the demagnetizing energy $2 \pi M_{S}^{2}$ so that $K_{z} \approx 0$, and $K_{\perp}$ is mostly determined by a small $K_{y}$. For $Q>1$, since the hard axis anisotropy is $K_{\perp}$ $=\left|K_{y}-K_{x}\right|$ which is independent of PMA, $u_{C}$ remains mostly invariant with $Q$.

Even if a DW does not go into continuous motion, it can still be displaced over some distance by a current less than $u_{C}\left(\sim J_{C}\right) .{ }^{3,4}$ Figure 3(a) shows that at $u=20 \mathrm{~m} / \mathrm{s}$ (less than $u_{C}$ for all samples), DWs in samples with $Q \approx 1$ can be displaced by as much as $\sim 100 \mathrm{~nm}$ in $\sim 10 \mathrm{~ns}$. Such DW displacement as a function of current below the continuous motion threshold is shown in Fig. 3(b), and the displacement attained per unit of current (slope of each fitted line) is defined as the "adiabatic displacement efficiency." When the
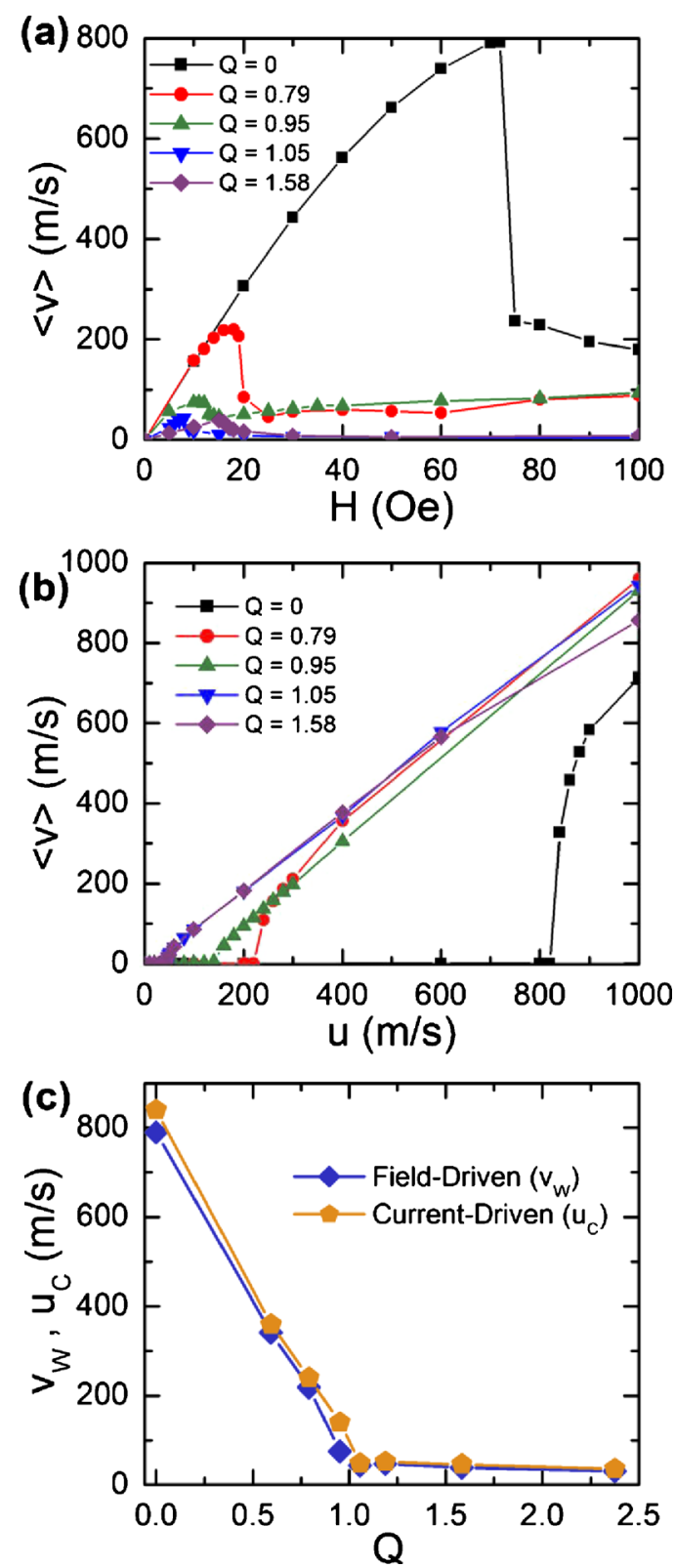

FIG. 2. (Color online) Average DW velocity versus (a) applied magnetic field and (b) normalized current. (c) Dependence of DW velocity at the Walker breakdown $v_{W}$ (field-driven case) and critical current for steady DW motion $u_{C}$ (current-driven case) on PMA.

demagnetizing field in the strip is compensated by the built-in perpendicular anisotropy field $(Q \approx 1)$, the DW is "softened" and can be displaced adiabatically with greater efficiency [Fig. 3(c)].

In a perfect strip with no extrinsic pinning, this DW displacement is "adiabatic" in the sense that the DW springs back reversibly to its initial position when the applied current is turned off. ${ }^{4}$ However, in a strip with a nonuniform pinning potential landscape, the displaced DW can fall into the nearest pinning potential well when the current is turned off. Thus, in a real strip with engineered notches or randomly dispersed defects in a sputtered structure, a soft DW may effectively hop from one pinning site to another by adiabatic spin transfer torque. While our simulations were performed at zero temperature, this current-driven displacement of soft DWs is expected to be more efficient at finite temperature, ${ }^{10,21}$ as DW depinning is a thermally activated process. 

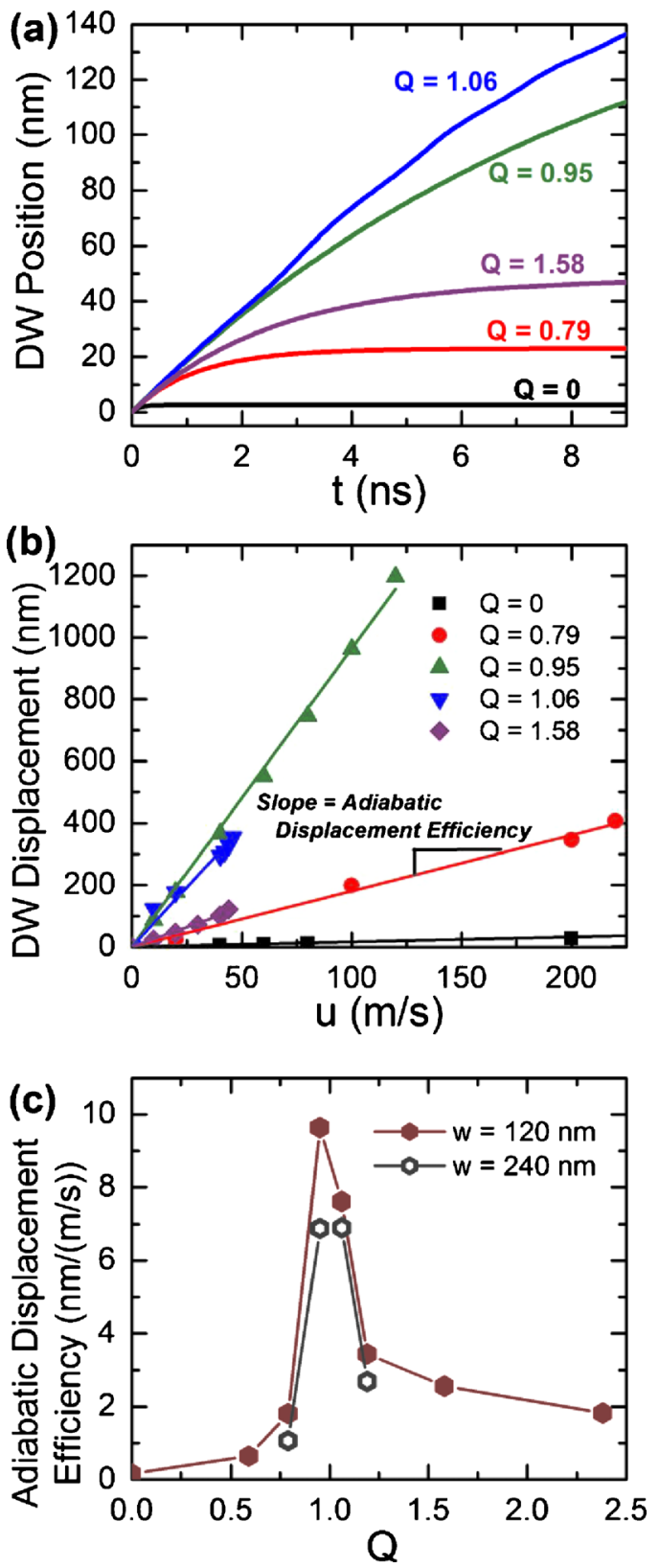

FIG. 3. (Color online) (a) DW position as a function of time at $u$ $=20 \mathrm{~m} / \mathrm{s}$, which is less than $u_{C}$. The $Q=0.95$ and $Q=1.06$ curves saturate at about $170 \mathrm{~nm}$ and $180 \mathrm{~nm}$, respectively, after $25 \mathrm{~ns}$. (b) DW displacement as a function of current below $u_{C}$. (c) Dependence of DW adiabatic displacement efficiency on PMA. Data for a wider strip (width $=240 \mathrm{~nm}$ ) are shown as well for reference.

Using patterned multilayer films (such as $\mathrm{Co} / \mathrm{Pt}$ ) with $Q \approx 1$ may be a practical means of enhancing currentinduced DW motion in devices. It is straightforward to tune the PMA of multilayer films by systematically adjusting the thicknesses of individual layers with an accuracy of $<1 \AA$ using evaporative or sputtering deposition. The adiabatic displacements of transverse/Bloch DWs for strips with different widths are similarly enhanced at $Q \approx 1$ [Fig. 3(c)], showing the applicability of tuning PMA for strips of various dimensions. For device applications requiring manipulation of multiple DWs along a magnetic track, ${ }^{7}$ it may be desirable to use a strip with $Q \geq 1$ that contains simple Bloch DWs [e.g., $Q$ $=1.06$, Fig. $1(\mathrm{~d})]$, rather than $Q \leq 1$ that may exhibit wide transverse DWs and complex magnetization patterns [e.g., $Q=0.95$, Fig. 1(c)]. In an engineered out-of-plane magnetized strip with PMA just strong enough to prevent spontaneous formation of stripe domains, a series of soft Bloch DWs may be initialized controllably and moved efficiently with small currents. In addition to the possible device applications, by experimentally measuring current-induced DW displacement for samples with a variety of PMA quality factors and engineered pinning sites, the fundamental physics of spin transfer torque may be understood more clearly.

This work was supported by the NSF Graduate Research Fellowship. S.E. thanks Mark Mascaro and Michael Donahue for their help on OOMMF.

${ }^{1}$ L. Berger, J. Appl. Phys. 55, 1954 (1984).

${ }^{2}$ G. Tatara and H. Kohno, Phys. Rev. Lett. 92, 086601 (2004).

${ }^{3}$ Z. Li and S. Zhang, Phys. Rev. Lett. 92, 207203 (2004).

${ }^{4}$ G. Beach, M. Tsoi, and J. Erskine, J. Magn. Magn. Mater. 320, 1272 (2008)

${ }^{5}$ A. Thiaville, Y. Nakatani, J. Miltat, and Y. Suzuki, Europhys. Lett. 69, 990 (2005).

${ }^{6}$ S. Fukami, Y. Nakatani, T. Suzuki, K. Nagahara, N. Ohshima, and N. Ishiwata, Appl. Phys. Lett. 95, 232504 (2009).

${ }^{7}$ S. S. P. Parkin, M. Hayashi, and L. Thomas, Science 320, 190 (2008).

${ }^{8}$ D. A. Allwood, G. Xiong, C. C. Faulkner, D. Atkinson, D. Petit, and R. P. Cowburn, Science 309, 1688 (2005).

${ }^{9}$ A. Yamaguchi, K. Yano, H. Tanigawa, S. Kasai, and T. Ono, Jpn. J. Appl. Phys., Part 1 45, 3850 (2006).

${ }^{10}$ S. Seo, K. Lee, W. Kim, and T. Lee, Appl. Phys. Lett. 90, 252508 (2007).

${ }^{11}$ S. Jung, W. Kim, T. Lee, K. Lee, and H. Lee, Appl. Phys. Lett. 92, 202508 (2008)

${ }^{12}$ S. Fukami, T. Suzuki, N. Ohshima, K. Nagahara, and N. Ishiwata, J. Appl. Phys. 103, $07 \mathrm{E} 718$ (2008).

${ }^{13}$ D. Ravelosona, D. Lacour, J. A. Katine, B. D. Terris, and C. Chappert, Phys. Rev. Lett. 95, 117203 (2005).

${ }^{14}$ M. Yan, A. Kákay, S. Gliga, and R. Hertel, Phys. Rev. Lett. 104, 057201 (2010)

${ }^{15}$ O. A. Tretiakov and A. Abanov, Phys. Rev. Lett. 105, 157201 (2010).

${ }^{16}$ C. Lin, G. Gorman, C. Lee, R. Farrow, E. Marinero, H. Do, H. Notarys, and C. Chien, J. Magn. Magn. Mater. 93, 194 (1991).

${ }^{17}$ M. J. Donahue, http://math.nist.gov/oommf/.

${ }^{18}$ A. P. Malozemoff and J. C. Slonczewski, Magnetic Domain Walls in Bubble Materials (Academic, New York, NY, 1979).

${ }^{19} \mathrm{R}$. Allenspach, http://www.zurich.ibm.com/st/magnetism/ spintevolve.html.

${ }^{20} u=\left(g \mu_{B} P J / 2 e M_{S}\right)$ where $P$ is the spin polarization and $J$ is the current density. For Co, assuming $g=2, u \approx\left(4 \times 10^{-11} \mathrm{~m}^{3} / \mathrm{A} \mathrm{s}\right) P J$.

${ }^{21}$ E. Martinez, L. Lopez-Diaz, O. Alejos, and L. Torres, J. Appl. Phys. 106, 043914 (2009). 